\title{
Metrology concept design of the GAIA basic angle monitoring system
}

\author{
Mariëlle van Veggel ${ }^{*}$, Rob Vink ${ }^{\mathrm{b}}$, Nick Rosielle ${ }^{\mathrm{a}}$, Henk Nijmeijer ${ }^{\mathrm{a}}$, Arno Wielders ${ }^{\mathrm{b}}$ \\ ${ }^{\mathrm{a}}$ Technische Universiteit Eindhoven, Department of Mechanical Engineering, P.O. Box 513, \\ 5600 MB Eindhoven, The Netherlands; \\ ${ }^{\mathrm{b}}$ TNO TPD, Division of Optical Instrumentation, Stieltjesweg 1, 2628 CK Delft, The Netherlands
}

\begin{abstract}
The GAIA satellite, scheduled for launch in 2010, will make a highly accurate map of our Galaxy. It will measure the position of stars with an accuracy of 50 prad using two telescopes, which are positioned under a 'basic' angle between the the lines-of-sight of the telescopes of $106^{\circ}$. With a Basic Angle Monitoring system, variations of this angle will be measured with 5 prad accuracy, to correct for these variations on the measured position of stars.

A conceptual design of the Basic Angle Monitoring system is presented. Two pairs of parallel laser bundles are sent to the telescopes, which create two interference patterns. If the basic angle varies, the interference patterns will shift. The optical design is such that the rotation of one pair of beams with respect to the other pair, does not affect the measured basic angle.

The position stability requirement of the mirrors is a maximum shift of $1 \mathrm{pm}$ in 6 hours. For material stability and good thermal and mechanical properties, Silicon Carbide has been chosen. The structural design is such that the design is as much monolithic as possible. The alignment is performed along the horizontal plane with external and removable alignment mechanisms. The components are locked by adhesives.
\end{abstract}

Keywords: metrology, interferometer, GAIA, basic angle monitoring

\section{INTRODUCTION}

In astronomy large interest exists to find out more about the origin and evolution of the universe. As well as studies involving watching background radiation at distances of more than 12 billion light-years, scientists are also very interested in the evolution of the Milky Way. As a successor of the Hipparcos mission, ESA is currently preparing a satellite called GAIA (Global Astrometric Interferometer for Astrophysics). GAIA will utilize the same parallax measuring principle as Hipparcos, but with increased accuracy. It will be capable of measuring the position and velocity of a billion stars in 6 years with a positional accuracy of 50 prad and a velocity accuracy of $1 \mathrm{~km} / \mathrm{s}$ at magnitudes as low as 15 . It will also be capable of measuring objects down to magnitude 20, but at lower accuracy.

The GAIA satellite (Fig. 1) consists of three modules: the sun shield, the service module (SVM) and the payload module (PLM). The function of the sun shield is to protect the SVM and the PLM from direct sunlight, because the solar radiation and light would have negative influence on the instruments. The sunshield has a diameter of approximately 11 meters. In the centre, it contains a large solar panel with a diameter of $3 \mathrm{~m}$, which provides for the necessary energy. The SVM is a closed hexagonal box, containing service equipment like video processing units, antennas and small engines for steering the satellite. The payload module accommodates the scientific instrumentation (Fig. 2):

- Two identical astrometric telescopes, which measure the position and velocity of stars. The basic angle (the angle between the lines of sight between the telescopes) will be $106^{\circ}$.

- Focal plane assembly: a large CCD camera, which collects the light from the telescopes.

- Spectrometric instrument, which determines the radial velocity and the chemical composition of stars.

\footnotetext{
*a.a.v.veggel@tue.nl; phone ++31 (0)40 247 4925; fax ++31 (0)40 246 1418; www.tue.nl
} 


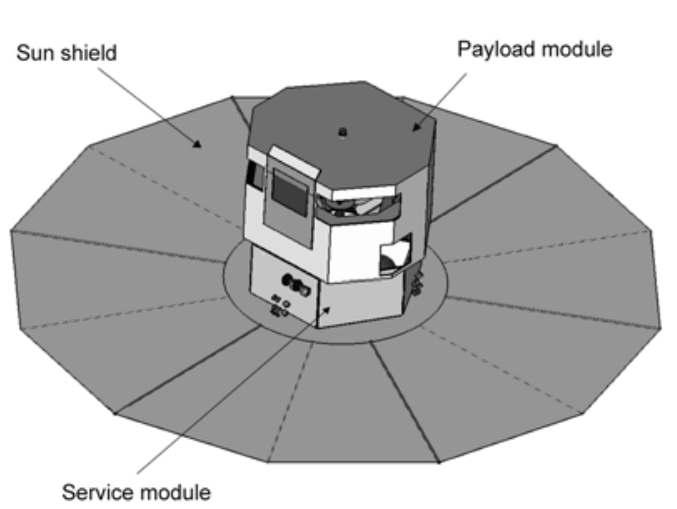

Fig. 1 Impression of the GAIA satellite ${ }^{1}$ (courtesy Astrium)

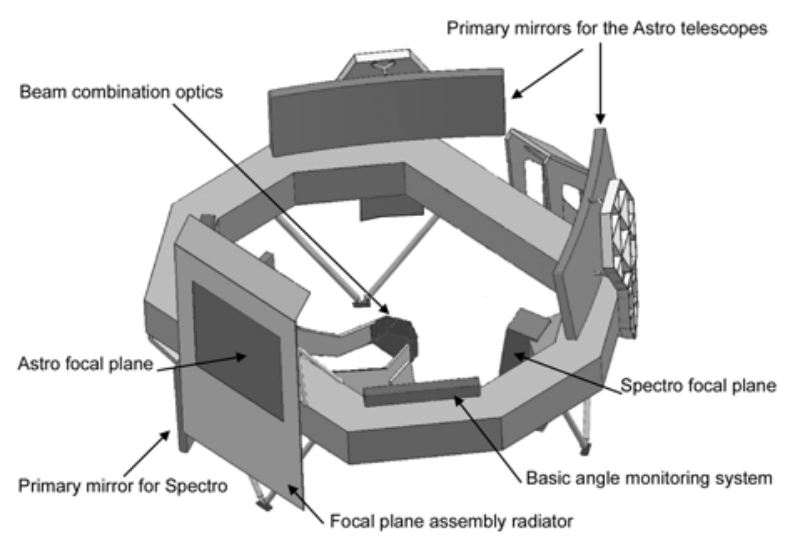

Fig. 2 Impression of the payload module of GAIA with scientific instruments ${ }^{1}$ (courtesy Astrium)

The scientific instruments are mounted on an octagonal optical bench in the preliminary design. To ensure mechanical and thermal stability in the PLM, it has been decoupled from the SVM as much as possible by placing the optical bench on three $\mathrm{V}$-shaped isostatic rods. The maximum diameter of the optical bench is $3 \mathrm{~m}$. The payload module will be covered with a multi-layer insulation tent providing insulation from external influences.

The satellite instruments will have to survive certain environmental conditions (pre-launch and launch), without damage and without losing alignment. On the ground, the satellite will be in air at ambient pressure of $10^{5} \mathrm{~Pa}$, at regular earth temperature variations $(250 \mathrm{~K}$ to $310 \mathrm{~K})$ and in varying air humidity levels. For testing, some vibrations will be applied to the satellite. During launch the pressure will drop to $10^{-5} \mathrm{~Pa}$. During its first orbits around the Earth, temperature can vary from $150 \mathrm{~K}$ in the shade to $390 \mathrm{~K}$ in the sunlight. Launch vibrations are very severe, up to $45 \mathrm{~g}$. During operation at GAIA's final destination, the ambient pressure will be $10^{-10} \mathrm{~Pa}$. The temperature of the PLM will be $160 \mathrm{~K} \pm 30 \mathrm{mK}$. The main heat source in the PLM will be the focal plane. Vibrations only occur during periodic engine bursts. The satellite will rotate around its axis every 6 hours, which means the telescopes make a scanning movement with respect to the universe.

During operation, the scientific instruments not only need to stay aligned for 6 years, the scientific instruments also need to have a high stability level in the cycle time of 6 hours in order to reach the 50 prad accuracy of measured positions of stars. The PLM will be made almost completely of Silicon Carbide (SiC) for this reason, because it is a lightweight material $\left(\rho=3100 \mathrm{~kg} / \mathrm{m}^{3}\right)$, it is very stiff $(E=420 \mathrm{GPa})$, it has low thermal expansion $\left(\alpha=2 \cdot 10^{-6} / \mathrm{K}\right)$ and it has high thermal conductivity $(\lambda=170 \mathrm{~W} / \mathrm{m} / \mathrm{K})$. It is also a very stable material.

To be able to link the measured positions of stars of one telescope, to the measured positions of stars of the other telescope, it is very important to know the angle between both telescopes. This "basic angle" is measured prior to launch. Maximum variations of 15 prad are expected on the basic angle in the 6-hour cycle. However, this expected variation is very small and may be larger. Therefore, the satellite will be equipped with a metrology system, which will specifically measure the variations of the basic angle with an accuracy of 5 prad, with the goal of mathematically correcting for these variations in the measured positions of stars. The full name of this metrology system is the Basic Angle Monitoring (BAM) system. The BAM system will also be made of SiC.

In this paper the conceptual design development of such BAM system will be discussed. In section 2 the optical design and requirements of the system will be discussed. Section 3 will deal with some design considerations. In section 4 , mechanical design concepts will then be presented. Finally, the paper will end with a discussion on future research.

\section{OPTICAL DESIGN AND REQUIREMENTS}

\subsection{Measurement principle}

Fig. 3 shows the top view of the measurement principle of the BAM system in GAIA. The octagonal structure, on which the scientific instruments are mounted, is visible. The primary mirrors of both telescopes are shown on the left and the 
right. BAM Bars 1 and 2, which are facing the primary mirrors of the telescope on the opposite side of the octagonal structure, contain the BAM system. Essentially, on BAM bar 1 a laser source and collimator send out a laser beam, which is split into two coherent laser beams on BAM bar 1. The light of each bundle is split again: one part is sent to astrometric telescope 1 and the other part is sent to BAM bar 2. This creates a pair of coherent and parallel bundles traveling through astrometric telescope 1 and a pair of coherent and parallel bundles traveling toward BAM bar 2. The bundles arriving at BAM bar 2 will be redirected toward astrometric telescope 2. Each bundle pair will finally interfere like double slit refraction creating two interference patterns on the focal assembly (Fig. 4).

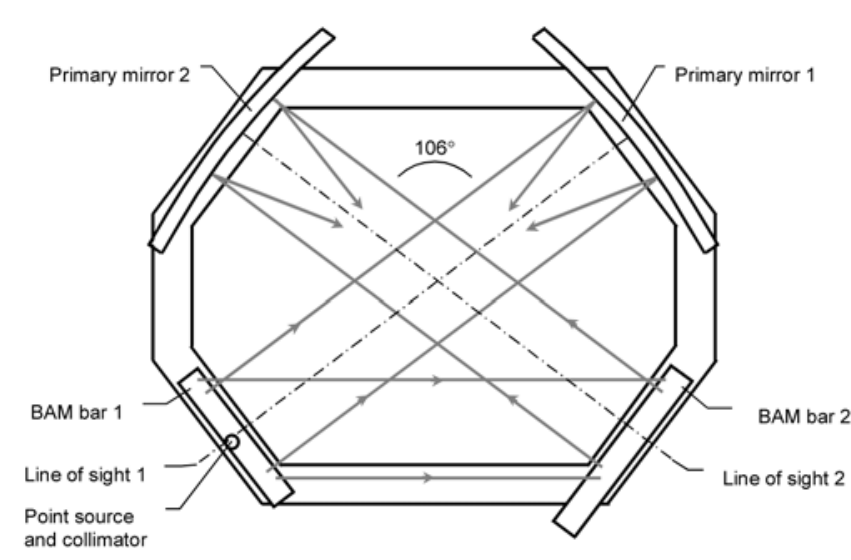

Fig. 3 Measurement principle of the Basic Angle Monitoring system

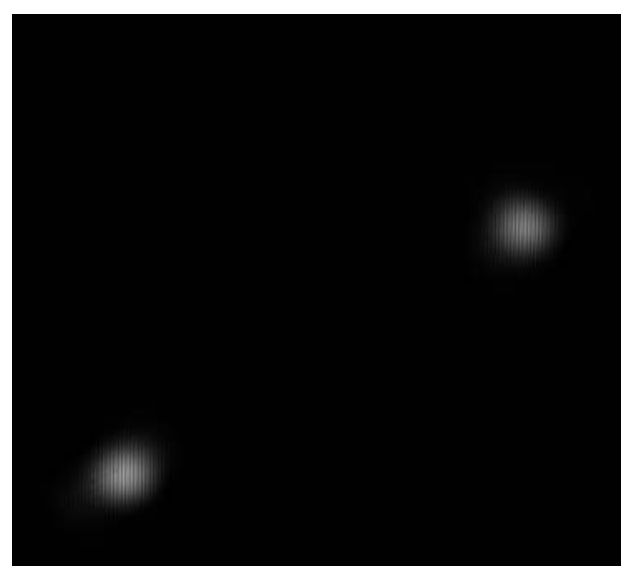

Fig. 4 The interference patterns of two pairs of interfering laser beams

If one of the fringe patterns shifts with respect to the other fringe pattern, this is a measure for the change in angle between the telescopes. To be able to reach the $10 \mu$ as accuracy in star position measurements, it is necessary to measure the angle variation between both telescopes with an accuracy of $1 \mu$ as. A small rotation of the primary mirror of the telescope, causes the fringe pattern to shift. The BAM subsystems bar 1 and bar 2 should not affect the Line-ofSight (LOS) measurement. Any rigid body movement should have no influence on the differential fringe pattern shift.

\subsection{Optical design of the BAM bars}

The optical design of the BAM bars will be very similar to the optical design of the bars of the GAIA OPD testbench, which has been developed in 1999 at TNO TPD in cooperation with Astrium France, to prove the measurement principle $^{3}$ (Fig. 5 and Fig. 6). The main differences between the GAIA OPD testbench and the BAM system for GAIA, are: the angle between the lines-of-sight of the BAM bars, which is $0^{\circ}$ for the testbench and $106^{\circ}$ for the BAM system, the size of the system, the fact that the GAIA OPD testbench was made largely of aluminium and lifetime. The baselength between two parallel bundles for the GAIA OPD testbench is $93 \mathrm{~mm}$, whereas the baselength of the BAM system will be $532 \mathrm{~mm}$. The optical design of BAM system is such that rigid body translations and rotations do not affect the OPD of the BAM system and thus the fringe position does not change for these rigid body movements.

The angular size of one fringe should be equal to the angular size of the diffraction pattern of a star. The wavelength in which GAIA will operate is $380-780 \mathrm{~nm}$, the center wavelength $(\lambda)$ of $580 \mathrm{~nm}$ is used for calculations. From this wavelength and with $W(=1.4 \mathrm{~m})$, the width of the telescope primary mirror the angular size of the star diffraction pattern can be calculated:

$$
S=\frac{2.44 \cdot \lambda}{W}=1.0 \mu \mathrm{rad}
$$

The needed number of fringes over the metrology diffraction spot $(N \approx 60)$ and the focal length of the telescope $(f=47$ $\mathrm{m})$, determine the needed diffraction spot size of the interference pattern $\left(D_{\text {Airy }}\right)$ and thus the beam diameter $(D)$.

$$
\begin{aligned}
& D_{\text {Airy }}=N \cdot S \cdot f=2.8 \mathrm{~mm} \\
& D=\frac{2.44 \cdot \lambda \cdot f}{D_{\text {Airy }}}=22 \mathrm{~mm} .
\end{aligned}
$$




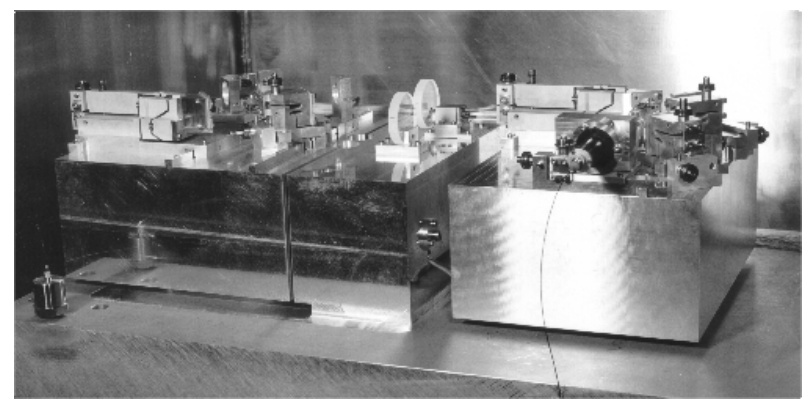

Fig. 5 BAM bars of the GAIA OPD Testbench

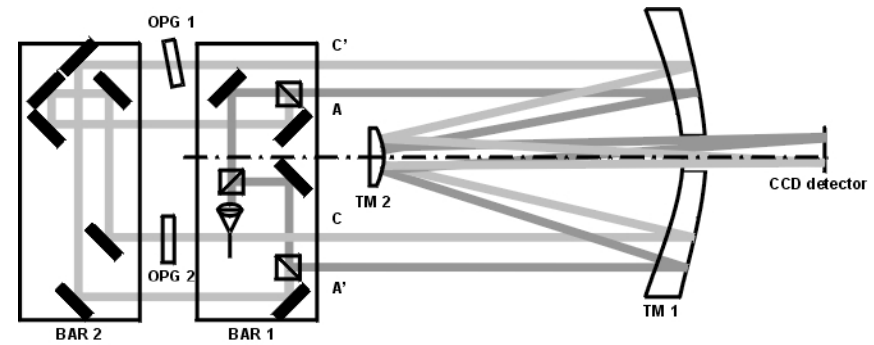

Fig. 6 Optical design of the GAIA OPD Testbench

\subsection{Alignment and stability requirements}

The resolution of the LOS measurement should be 5 prad, being 1/190,000 times the diffraction limit. To this end, the optical components must reach certain alignment and stability requirements. These requirements can be subdivided in position and angle requirements.

\subsubsection{Position alignment and stability}

The position alignment and stability of the optical components is determined by the fringe stability. The fringe stability again, depends on Optical Path Difference (OPD) alignment and stability and wavelength stability. The OPD is defined here as the difference in distance both beams have traveled from the source towards the focal plane.

\section{OPD alignment}

The white fringe of the interference pattern should preferably be in the centre of the diffraction spot. If a decenter of the white fringe of 10 fringes is allowed, the initial OPD is allowed to be $5 \mu \mathrm{m}$. A displacement of an optical component of $1 \mathrm{~mm}$, causes, in the worst case, a $2 \mathrm{~mm}$ OPD. Therefore, the maximum total misalignment of the optical components may be $2.5 \mu \mathrm{m}$. Since the optical design consists of about 6 mirrors maximum for one branch, this provides, assuming a normal distribution of position accuracy of the individual optical components, for the necessary position accuracy of individual components:

$$
\delta x=\frac{2.5}{\sqrt{6}}=1 \mu \mathrm{m} .
$$

\section{OPD stability}

The interfringe distance can be expressed in OPD of the BAM, which equals one wavelength of OPD, thus $532 \mathrm{~nm}$. Now the $5 \operatorname{prad}\left(\theta_{\text {acc }}\right)$ measurement accuracy can be calculated to an OPD-stability in 6 hours:

$$
\delta O P D=\frac{\lambda \cdot \theta_{a c c}}{S}=\frac{532 \cdot 10^{-9} \cdot 5 \cdot 10^{-12}}{1.0 \cdot 10^{-6}}=2.7 \mathrm{pm} .
$$

This means that a $2.7 \mathrm{pm}$ change in OPD will be interpreted as 5 prad change of LOS. The required OPD stability of the BAM system thus should be better than 2.7 pm over one revolution of 6 hours.

\section{Wavelength stability}

In case the optical path difference between interfering beams is not exactly zero, wavelength change results in fringe position change. The zero optical difference fringe (white fringe) should be well within the diffraction spot. When the white fringe is centered exactly in the diffraction spot, a wavelength change results in "breathing" of the fringes where the white fringe position is fixed. When the white fringe is decentered in the diffraction spot, a wavelength change results in an average fringe shift. The total number of fringes $N$ equals 60 . When the decenter in number of fringes of the white fringe equals $N_{d}=10$ (like stated above), an asymmetric change of the fringe pattern results from a wavelength change. In worst case the outermost fringe position is taken for calculations. Thus, when the wavelength change equals $\delta \lambda$ the shift of this fringe $(\delta O P D)$ equals: 


$$
\delta O P D=N_{d} \cdot \delta \lambda
$$

As calculated above, a $\delta O P D$ of $2.7 \mathrm{pm}$ corresponds to 5 prad LOS. When the error due to wavelength instability is allowed to be $0.5 \mathrm{pm}$, the maximal wavelength change should be:

$$
\delta \lambda=\frac{\delta O P D}{N_{d}}=\frac{0.5 \cdot 10^{-12}}{10}=0.5 \cdot 10^{-13} \mathrm{~m}
$$

Concluding, an initial OPD of $5 \mu \mathrm{m}$ combined with a wavelength stability $\delta \lambda / \lambda$ of $9 \cdot 10^{-8}$ is required for a lifetime of 6 years.

\subsubsection{Angle alignment and stability}

Two overlapping spots generate the fringe pattern. The number of fringes depends on the amount of overlap. For the OPD testbench ${ }^{3}$ an overlap requirement of $90 \%$ has been taken. For GAIA the overlap requirement is expected to be less than $90 \%$, but this value still is taken for calculations. Since the diffraction size of the Airy disc equals $60 \mu \mathrm{rad}$, the angular stability of one beam with respect to the other, equals $6 \mu \mathrm{rad}$. The optical design consists of about 6 mirrors maximum for one branch, leading to an angular accuracy for the reflected beam angle of:

$$
\delta \theta=\frac{6}{\sqrt{6}}=2.4 \mu \mathrm{rad} \text { stability requirement. }
$$

The angular alignment of the optical components has to be a factor of 2 better, due to reflection. Thus the alignment requirement for each mirror equals $1.2 \mu \mathrm{rad}$. When there is a linear angular drift of $1.2 \mu \mathrm{rad}$ over the complete 6 year mission, the angle stability of individual components over 6 hours should be better than 0.14 nrad over 6 hours.

The requirements stated above, are summarized in Table 1.

Table 1 Alignment and stability requirements of individual optical

Table 1 Alignment and stability requirements of individual optical
\begin{tabular}{|l|l|l|}
\hline Parameter for one component & Alignment requirement over 6 years & Stability requirement over 6 hours \\
\hline Position & $1 \mu \mathrm{m}$ & $1.1 \mathrm{pm}$ \\
\hline Angle stability & $1.2 \mu \mathrm{rad}$ & $0.14 \mathrm{nrad}$ \\
\hline
\end{tabular}

\section{DESIGN CONSIDERATIONS}

\subsection{Stability}

The stability requirements on the BAM system can be viewed in the scope of two different time periods:

1. Alignment of individual optical components with respect to each other during service life (6 years), including handling on Earth and launch, in the order of micrometers.

2. Positional and angular stability of individual optical components with respect to each other in a time period of 6 hours (in which the satellite rotates around its axis) in the order of $1 \mathrm{pm}$.

Especially the 'short' period stability requirements are extremely stringent. The position of the individual mirrors and beamsplitters will have to be stable to the picometer level over a period of 6 hours. Stability at nanometer level of a period of several hours has been reported before ${ }^{6}$. However, picometer stability has not been shown before. The stability of the design of the BAM system therefore needs special attention.

Dimensional stability can be viewed from the perspectives: material stability, structural stability and environmental stability. According to Marschall ${ }^{7}$ material stability means that mechanical and physical properties and dimensions of the component remain constant throughout its service life. Structural stability means that the (angular) position of one component in a device with respect to another component remains constant throughout a certain period.

Temporary or permanent changes in physical properties and dimensions can occur in three different circumstances:

1. A dimensional change occurring as a function of time in a fixed environment. 
2. A dimension change, measured under a fixed environment, after exposure to a variable environment.

3. A dimensional change, measured under a fixed environment and depending upon the environmental path used to reach the fixed environment.

The circumstances stated above do not include the circumstance in which a periodic dimensional change occurs if small environmental changes occur in that same period. E.g. when the temperature changes, thermal expansion of the material occurs. Such environmental instability can influence measurements, because dimensions of the metrology system change. Such dimensional changes are reversible and therefore not indicated as instability. However, if such changes cause the measurement to become less accurate, this could be seen as instability of the measurement and should be taken into account in the design of the structure.

In the case of the BAM system, dimensional instability can be caused by:

1. Externally applied stress: the component is exposed to mechanically applied loads.

2. Internal stress or a variation of internal stress. Internal stresses are stresses, which exist in a body in the absence of external forces.

3. Thermal influences: change in temperature or temperature gradient.

4. Radiation: especially solar radiation.

\subsection{Silicon Carbide}

In the introduction, it was explained that $\mathrm{SiC}$ has been selected as the material of which the PLM will be constructed, because of its good thermal and mechanical properties. In Table 2 the general characteristics of two types of SiC (sintered $\mathrm{SiC}(\mathrm{SSiC})$ and Carbon felt infiltrated $\mathrm{SiC}(\mathrm{C} / \mathrm{SiC})$ ) are shown in comparison to other ceramic materials and some metals. Besides direct physical properties like density $\rho$, Young's modulus $E$, thermal expansion $\alpha$, thermal conductivity $\lambda$ and specific heat $C_{P}$, also some indirect physical properties are shown in the table. These are, specific stiffness $E / \rho$, insensitivity for thermal gradients $\lambda / \alpha$, thermal diffusivity $\lambda / \rho / C_{P}$ and thermal stability $\lambda / \alpha / \rho / C_{P}$. The indirect properties should preferably be as high as possible, to limit deformation on mechanical or thermal loading. Both types of $\mathrm{SiC}$ score high on the thermal properties. $\mathrm{SSiC}$ also scores very high on specific stiffness.

Table 2 Mechanical and thermal properties of selected materials

\begin{tabular}{|c|c|c|c|c|c|c|c|c|c|}
\hline Material & {$\left[\mathrm{g} / \mathrm{cm}^{3}\right]$} & [GPa] & {$[\mu \mathbf{m} / \mathbf{m} / \mathbf{K}]$} & {$[\mathbf{W} / \mathbf{m} / \mathbf{K}]$} & {$[\mathrm{J} / \mathrm{kg} / \mathrm{K}]$} & $\begin{array}{l}\frac{E}{\rho} \\
{\left[\mathrm{GPa} \cdot \mathrm{cm}^{3} / \mathrm{g}\right]}\end{array}$ & $\begin{array}{l}\frac{\lambda}{\alpha} \\
{[\mathbf{W} / \mu \mathbf{m}]}\end{array}$ & $\begin{array}{c}\frac{\lambda}{\rho \cdot C_{P}} \\
{\left[\mathrm{~mm}^{2} / \mathbf{s}\right]}\end{array}$ & $\begin{array}{l}\frac{\lambda}{\alpha \cdot \rho \cdot C_{P}} \\
{\left[\mathbf{m}^{2} \mathbf{K} / \mathbf{s}\right]}\end{array}$ \\
\hline$\overline{\mathrm{SSiC}}$ & 3.1 & 420 & 2.5 & 180 & 680 & 135 & 72.0 & 85.4 & 34.2 \\
\hline $\mathrm{C} / \mathrm{SiC}$ & 2.6 & 248 & 1.2 & 170 & 670 & 61 & 141.7 & 93.6 & 78.0 \\
\hline $\mathrm{Al}_{2} \mathrm{O}_{3}$ & 3.9 & 320 & 7.0 & 28 & 880 & 82 & 4.0 & 8.4 & 1.2 \\
\hline $\mathrm{B}_{4} \mathrm{C}$ & 2.5 & 450 & 5.0 & 45 & 950 & 180 & 9.0 & 18.9 & 3.8 \\
\hline $\mathrm{Si}_{3} \mathrm{~N}_{4}$ & 3.2 & 320 & 3.2 & 35 & 680 & 100 & 10.9 & 16.1 & 5.0 \\
\hline $\mathrm{Be}$ & 1.8 & 287 & 11.4 & 190 & 1780 & 159 & 16.7 & 57.7 & 5.1 \\
\hline Al alloy & 2.7 & 70 & 24 & 220 & 880 & 26 & 9.2 & 92.6 & 3.9 \\
\hline Steel 316 & 7.8 & 205 & 17 & 45 & 480 & 26 & 2.6 & 12.0 & 0.7 \\
\hline Invar & 8.1 & 140 & 2 & 16 & 500 & 17 & 8.0 & 4.0 & 2.0 \\
\hline TiAlV & 4.4 & 114 & 8.6 & 6.7 & 526 & 26 & 0.8 & 2.9 & 0.3 \\
\hline Zerodur & 2.5 & 91 & 0.05 & 1.64 & 821 & 36 & 32.8 & 0.80 & 16.0 \\
\hline
\end{tabular}

Although there are other types of $\mathrm{SiC}$ on the market, the $\mathrm{SSiC}$ and $\mathrm{C} / \mathrm{SiC}$ illustrated here are the most common and the manufacturing processes of other types of $\mathrm{SiC}$ are very similar to either the $\mathrm{SSiC}$ process or the $\mathrm{C} / \mathrm{SiC}$ process. $\mathrm{SSiC}$ is manufactured, like shown in Fig. 7. The SiC crystals in the powder have a cubic crystal structure. The green blank has chalk-like behavior and is machined into near net shape (the density of such a block is very homogeneous, but the density of individual blocks can vary slightly. The machined green blank is sintered in an inert atmosphere at $2370 \mathrm{~K}$. An extremely homogeneous shrinkage of $17 \%$ occurs. Dimensions can be predicted with $0.4 \%$ accuracy, caused by the slight variation of density for individual blocks. 


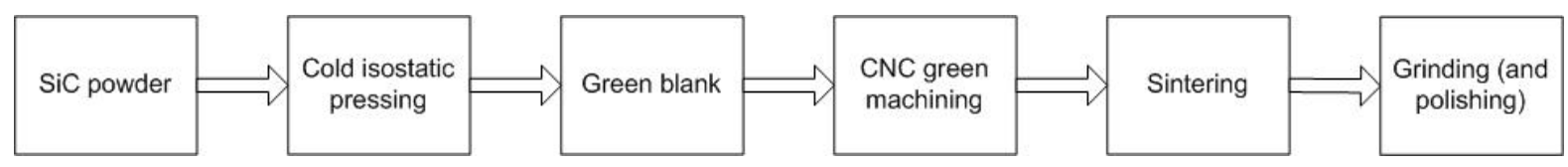

Fig. 7 A flowchart of the SSiC manufacturing process

The $\mathrm{C} / \mathrm{SiC}$ is produced in a slightly different process, like shown in Fig. 8. The graphite felt has very fine, open porous structure. The $\mathrm{SiC}$ conversion takes place by infiltrating the graphite at $1970 \mathrm{~K}$ with silicon carrying gases, like silicon monoxide, at which a reaction to $\mathrm{SiC}$ (crystals with a hexagonal crystal structure) takes place. No shrinkage occurs. However shape changes take place and dimensions can be predicted with $1 \%$ accuracy. The infiltration can be performed to a depth of $\sim 6 \mathrm{~mm}$. Because of the residual porosity of ca. $20 \%$, the $\mathrm{C} / \mathrm{SiC}$ part is generally coated with CVD SiC.

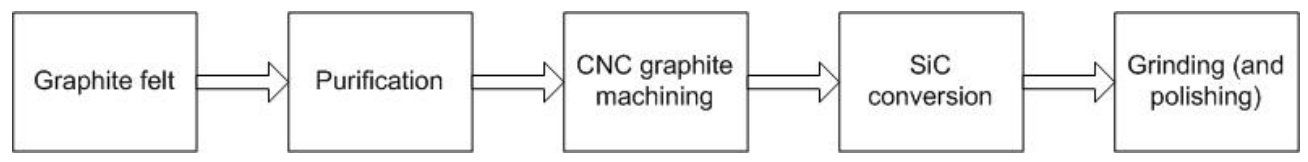

Fig. 8 A flowchart of the $\mathrm{C} / \mathrm{SiC}$ manufacturing process

Besides the fact that $\mathrm{SiC}$ is a ceramic, and thus has different manufacturing methods from metals, it also has some other challenges, especially with respect to final processing:

- Machining of $\mathrm{SiC}$ is not possible due to its hardness. Therefore, machining to near-net shape is usually performed in the green state of the material. Also due to its hardness, final processing (polishing, lapping, grinding, drilling, cutting) of $\mathrm{SiC}$ is a lengthy process and high quality diamond abrasives are necessary. Therefore, these processes are generally expensive. It is important to minimize the need for final processing as much as possible. In metalceramics bonding, dimensional tolerancing should be compensated for in the metal part, because this is easier to process.

- Because of the brittleness of $\mathrm{SiC}$, final processing is not trivial. Good attention has to be paid to the clamping of the $\mathrm{SiC}$ piece, limiting peak stresses as much as possible. The design of sharp edges should be avoided, certainly at locations on the product, which are mechanically loaded.

- $\quad \mathrm{SiC}$ is much stronger in compression than in tensile loading. Therefore, the locations, which are loaded, should be designed as much as possible under compressive loading or bending and as little as possible under tension.

\section{MECHANICAL DESIGN}

The mechanical design of the BAM system is still in its conceptual stage. Therefore, some concepts of the different elements of the BAM system discussed and the considerations of each concept will be discussed as well. The discussion will be ended with a set of possible conceptual designs. The BAM system can be divided into several elements that are considered, which are illustrated in Fig. 9.

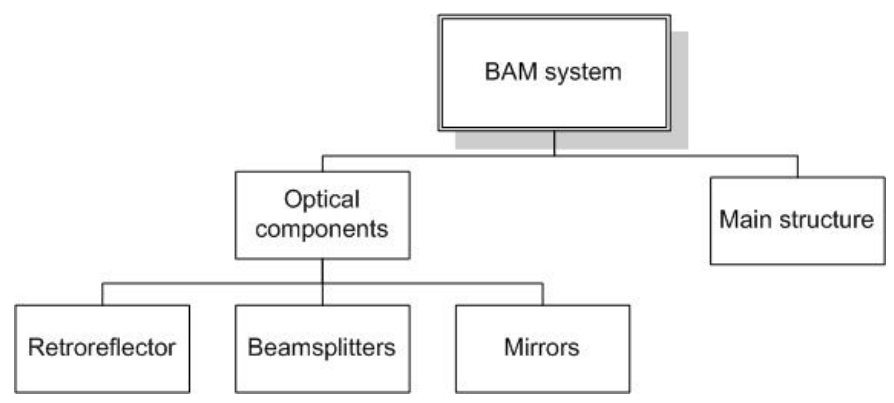

Fig. 9 Tree diagram of components in the BAM system 


\subsection{Main structure}

In the design of the main structure three basic options can be used in order to construct a main structure on which the optical components are mounted:

1. A non-modular option: a part of the optical bench of the payload module is also used as optical bench for the BAM system (Fig. 10).

2. A modular option: e.g. a rectangular box suspended on three leaf springs with elastic hinges (Fig. 11). The optical components are mounted in the box, or; a honeycomb plate on which the optical components are mounted, or; a cylinder suspended on three leaf springs with elastic hinges. The optical components are mounted in the box.

3. A partly modular option: e.g. each bar is divided into three groups of two mirrors or beamsplitters (Fig. 12).

The main structure is located right in the field of view (FOV) of the telescope. The non-modular option has the advantage in that respect, because the main structure hinders the FOV as little as possible. Moreover, the octagonal ring of the optical bench can be as high as possible, improving the stiffness of the ring.

The total mass of the BAM system would be less for the nonmodular option than for the modular options. For space applications in particular, it is essential to design as lightweight as possible.

The fewer components, the better for stability reasons, because component interfaces mainly cause instability of measurements. In case the six parts of the partly modular option can be made monolithic (except for the beamsplitters), this option has the fewest components. It should be noted however, that monolithic parts in the partly modular option are extremely difficult to make, because polishing of mirrors under strange angles and positions with respect to each other is extremely difficult. In that case the non-modular option has the fewest components.

The thermal environment is most probably more severe in service for the non-modular option than for the modular options, because the modular options can be coupled to the rest of the system, so that the heat transfer is smoother. The mechanical environment of the modular options, especially during launch is much more severe than for the non-modular option. The relatively large mass, which is suspended in a relatively less stiff manner on the octagonal ring, has lower modal frequencies and higher modal amplitudes, than the components mounted directly on the optical bench of the PLM.

The modular option has the advantage, that other systems are not dependent on it. If the development goes wrong, the development of the optical bench can continue without any problem, whereas in the non-modular concept, the development of both systems is interdependent.

There is no real difference in the alignment steps of the optical components on each bar, for the different main structure options, final alignment steps (two mirrors on bar 2) always need to be performed on the complete structure. If the alignment would completely be performed on the final structure, less alignment steps would be necessary than in case of alignment partially at another location, because in that case the alignment step of aligning the bars with respect to the telescopes would also be necessary.

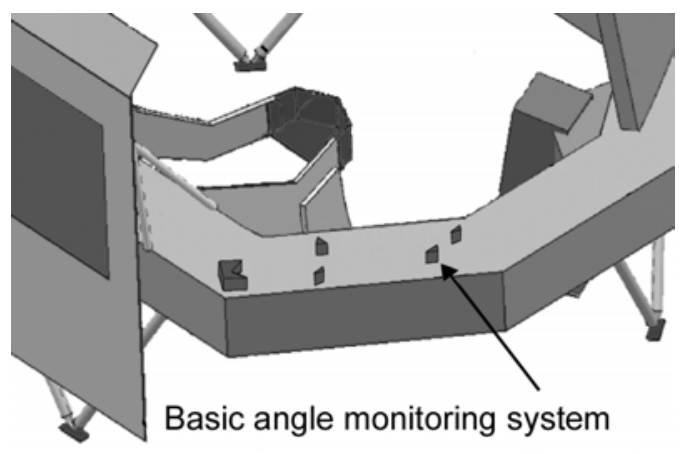

Fig. 10 Non-modular BAM system

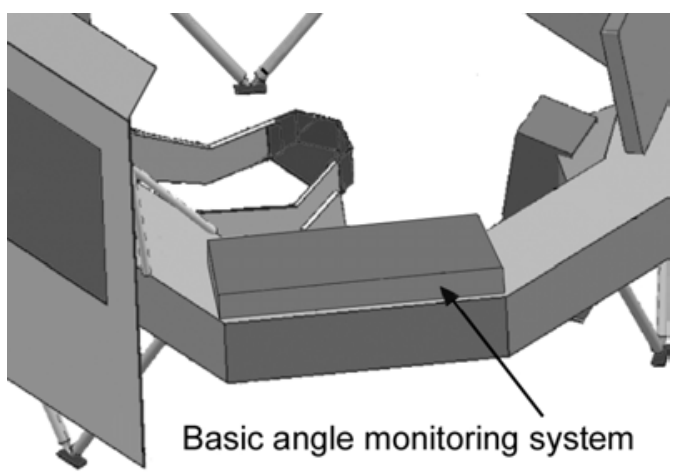

Fig. 11 Modular BAM system with optical components in a box

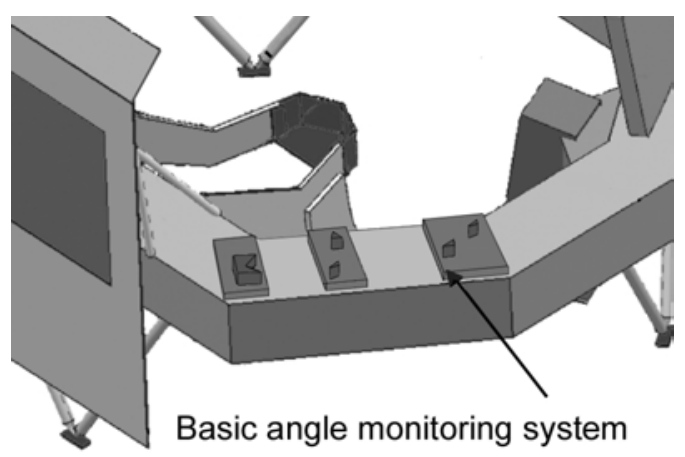

Fig. 12 Partly modular BAM system with grouped optical components 


\subsection{Optical components}

The design of the optical components of the BAM system is subdivided in mirrors, beamsplitters and the retroreflector. However, all have two common design aspects:

1. Mounting of the components on the optical bench.

2. Alignment methods and mechanisms.

\subsubsection{Mounting}

In mounting of optical components on the optical bench of the BAM system, essentially three basic options are considered:

1. Completely monolithic with optical bench (Fig. 13);

2. Connected to the optical bench in the horizontal plane (Fig. 14), and;

3. Connected to the optical bench in the vertical plane (Fig. 15).

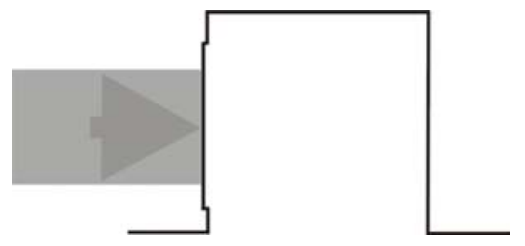

Fig. 13 Monolithic mounting

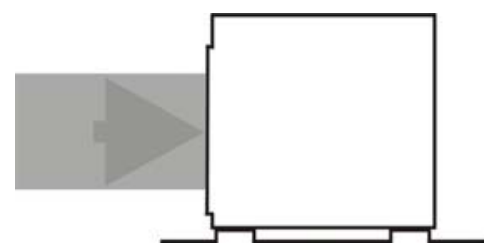

Fig. 14 Mounting on the horizontal plane

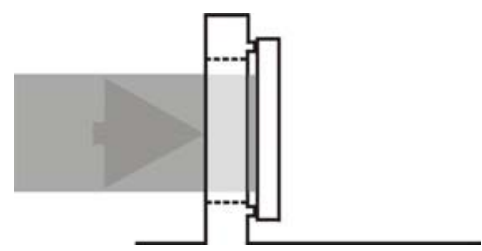

Fig. 15 Mounting on vertical plane

Monolithic mounting is better than non-monolithic mounting for stability reasons. Because Silicon Carbide itself is very stable, instability will mainly result from the instability of the structure. If the complete structure is monolithic, there are no joints and the complete stability will arise from the material stability.

However, monolithic mount will not be possible for the beamsplitters, because beamsplitters are made of a different material. Additionally, for polishing of the mirrors it is necessary to have a flat surface, which can be polished in the horizontal plane. It is very difficult to polish a surface, when some material protrudes in front of the mirror plane. In this argument it is more logical to choose a non-monolithic mounting, such that the mirror surface can be polished in the horizontal plane on a polishing bed.

Since the alignment is mainly performed along the horizontal plane, it seems a logical choice to mount the mirrors and beamsplitters along the horizontal plane. This argument is strengthened by the fact that the rough alignment of a vertical bracket would be possible with $50 \mu \mathrm{m}$ position accuracy, meaning that a large adjustment range would be necessary for fine alignment. However, in the case of beamsplitter mounting it is better to mount the beamsplitter at the reflective surface, because the position of that surface should remain stable. Because this surface is vertical, vertical mounting is the logical choice.

\subsubsection{Alignment methods}

With respect to the alignment methods, a distinction should be made between fine alignment methods and rough alignment methods. Fine alignment methods are:

1. Fine alignment based on elastic deformation (e.g. leaf springs and elastic hinges, Fig. 16).

2. Fine alignment achieved by polishing a certain surface until complete alignment is achieved (e.g. if a mirror disc is mounted onto a base via three raised areas and the alignment is achieved by polishing the raised areas).

3. Fine alignment achieved by designing an external alignment mechanism, which positions the mirror or beamsplitter and after which the mirror or beamsplitter is tensioned, brazed or glued onto the main structure (Fig. 17).

Rough alignment methods could be:

1. Components are monolithic with the main structure, which are located sufficiently accurate by machining the green shape. 
2. Rough alignment achieved by designing an external alignment mechanism, which positions the mirror or beamsplitter, after which the component is tensioned, brazed or glued onto the main structure (Fig. 17).

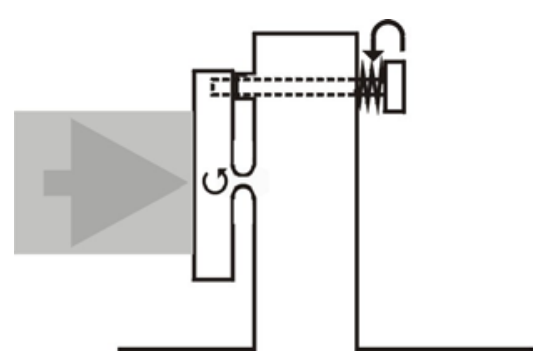

Fig. 16 Elastic fine alignment mechanism

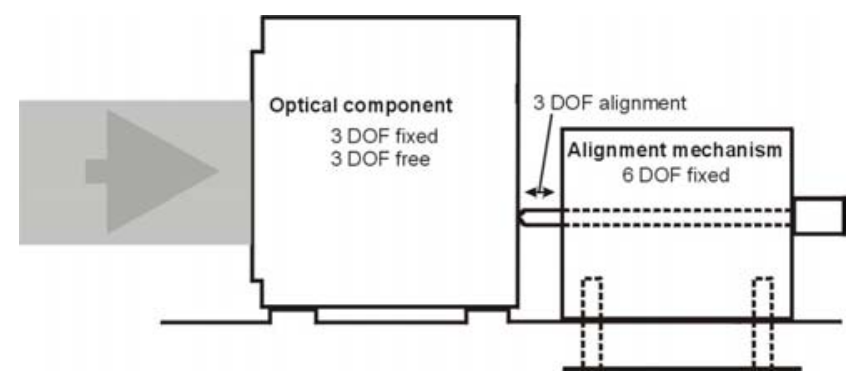

Fig. 17 Rough and fine external alignment

For alignment, stability is also an important issue. Because of the monolithic nature of an elastic hinge, stability would seem to be guaranteed. Introducing stress into any material will not improve the stability of a material. In alignment mechanisms based on elastic deformation however, stresses are introduced. An added difficulty is that the force, which needs to be applied to obtain alignment, should be such that the alignment remains stable.

In polishing surfaces for alignment, the additional assumption is made that two surfaces are mounted together and should remain at the same location with respect to each other. To achieve this, a sufficiently high normal force would need to be applied to the contact area. This means that stresses and strains are introduced into the contact area. To maintain alignment, the normal force would also need to be sufficiently stable and additionally, a mounting based on friction is a quasi-stable mounting, meaning that if the forces are too high, the bodies will shift with respect to one another and at lower force levels slip-fronts will enter the contact area, causing hysteresis.

Alignment with an external alignment mechanism offers the advantage that the alignment could be achieved without introducing stresses and strains into the optical components. However, this assumption should be modified slightly in the sense that the post-alignment fixation method might introduce stresses into the component. Fixation methods that are considered are tensioning, gluing and brazing.

\subsection{Conceptual designs}

The discussion above provides some insight in what the BAM system should globally look like. The main structure of the BAM system is the optical bench of the PLM. The 8 pieces of the optical bench are open back honeycomb boxes. On two of the pieces on the closed side some holes have been made, for fixation of the alignment mechanisms. The surfaces of the raised areas of the optical bench are polished, so that the flatness of the raised areas, also with respect to each other, is such that alignment along the horizontal axis in the reflective planes of the optical components is not necessary. The pieces are brazed together and the telescopes are installed. In the next step, the components of the BAM system are installed. External alignment mechanisms are placed and the mirrors and beamsplitters are placed. The alignment mechanisms are already positioned with an accuracy of $0.05 \mathrm{~mm}$, meaning that the rough alignment of the optical components is also $0.1 \mathrm{~mm}$. The fine alignment range of the mirrors and beamsplitters should be $0.1 \mathrm{~mm}$ with a resolution of $1 \mu \mathrm{m}$. The alignment mechanisms align the optical components until complete alignment has been obtained. Fixation of the optical components is then performed by injection of adhesive.

\subsubsection{Mirrors}

In one possible mirror design, the mirror is a cube, which rests on three raised areas of the optical bench. The plane, which rests on the notches, has been polished square with the mirror surface. The notches are surrounded with adhesive canals, through which the adhesive is ejected to fixate the mirrors on the optical bench (Fig. 18).

In the other design the fixation is performed by a mechanical tensioning connection (Fig. 19). 


\subsubsection{Beamsplitters}

The beamsplitters have two interfaces, in spite of the statement above, that as few as possible component interfaces is better for stability reasons. The beamsplitter itself is mounted onto a base using the polished raised areas method. The base is mounted onto the optical bench in the same manner as the mounting of the mirrors (Fig. 20).

\subsubsection{Retroreflector}

There are several different ways of making a retroreflector. One design is polishing both mirrors in one monolithic piece (Fig. 21), which is fixated to the optical bench the same way as the mirrors and beamsplitter base. The second option is to use two mirrors like shown above and align and fixate them with one alignment mechanism, which defines the $90^{\circ}$ angle between the mirror surfaces (Fig. 22). In the third option, a base is fixed to the optical bench. It also defines the $90^{\circ}$ angle between the two mirrors, which are mounted onto the base with the polished raised areas method (Fig. 23).

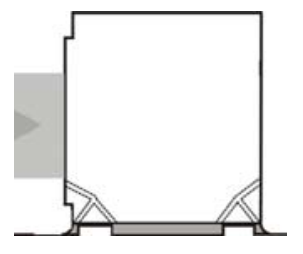

Fig. 18 Adhesive mounting

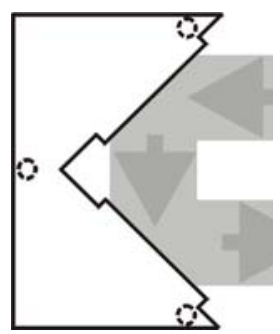

Fig. 21 Monolithic retroreflector

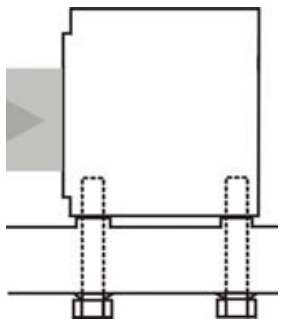

Fig. 19 Tensioned mounting

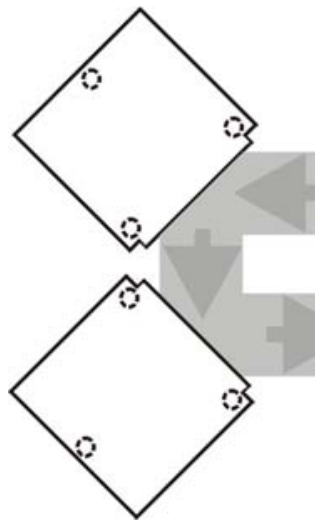

Fig. 22 Two-separatemirrors retroreflector

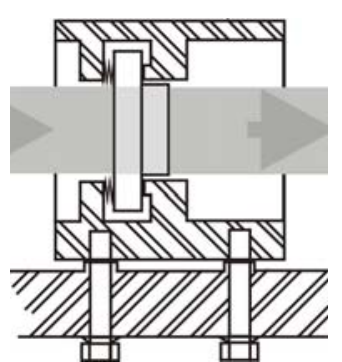

Fig. 20 Beamsplitter mounting

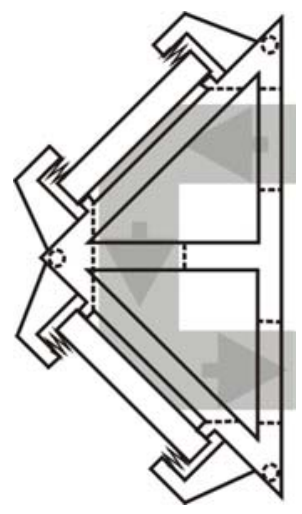

Fig. 23 Retroreflector with base and clamped mirrors

\section{CONCLUSIONS}

In this report, design considerations have been presented for the Basic Angle Monitoring system of GAIA, which will measure variations of the angle between the lines-of-sight of two telescopes with 5 prad accuracy. The optical design consists of two separate bars from which two parallel bundles travel toward the telescope and interfere at the focal plane. A variation of the angle between the lines-of-sight, causes a shift of one interference pattern with respect to the other. The optical design is such that the measured angle is insensitive for rotation of one of the bars with respect to the other. The optical components should be aligned with $1 \mu \mathrm{m}$ and $1.2 \mu \mathrm{rad}$ accuracy in 6 years of operation. The stability of the optical components over 6 hours cycle time should be $1.1 \mathrm{pm}$ and $0.14 \mathrm{nrad}$. This stability is determined by the environmental stability, material stability and the stability of component interfaces. Silicon Carbide is a material, which has such good thermal and mechanical properties that the stability requirements should be achievable. Silicon Carbide however, is very hard and needs special attention in the design process. Shaping should already be performed before sintering. Therefore, the shrinkage effects during sintering should be considered carefully. Because of the hardness of Silicon Carbide, final processing (grinding, polishing, etc.) should be limited to minimum. Some concepts have been presented of the design of optical components for the Basic Angle Monitoring system in GAIA. The components are mounted onto the optical bench of the PLM via a horizontal interface. The consideration of adhesive or mechanically fixing the components, needs yet to be made.

\section{FUTURE RESEARCH}

Currently, experiments are performed to test the mechanical behavior of small SiC tubes, which have been joined to another tube with several different techniques, like gluing, brazing and bolting. The goal of this experiment is to attain a first comparative impression of the stability of such joints in cyclic mechanical behavior. This will aid in the detail design of the optical components. 
The design of the BAM system will be developed further, to increasing detail, in order to be able to set up some experiments, which will test the stability and mechanical behavior of the designed mirrors, beamsplitters and retroreflector in an interferometer set-up. The goal is to have built a test set-up by the end of 2004.

\section{ACKNOWLEDGEMENTS}

This research was partly supported by the NIVR (Dutch Agency for Aerospace Programmes).

\section{REFERENCES}

1. http://sci.esa.int/science-e/www/area/index.cfm?fareaid=26

2. GAIA SLTRS project team. GAIA system level technical reassessment study final report. Astrium, France, 2002.

3. B. Snijders, P. Kappelhof, B.C. Braam, H.J.P. Vink, P. Verhoeff, B. Calvel, F. Safa, GAIA test-bench: monitoring the basic angle with microarcsecond accuracy. SPIE proceedings, vol. 4006, pp. 915-926

4. http://sci.esa.int/science-e/www/object/index.cfm?fobjectid=33883

5. F.Safa, GAIA Interferometric Basic Angle Monitoring in Orbit. Matra Marconi Space, internal report, 1998

6. N. van der Lee, P. Kappelhof, R. Hamelinck. Flexure-based alignment mechanisms: design, development and application. SPIE proceedings, vol. 5176, pp.94-107

7. Marschall, C.W., Maringer, R.E. Dimensional Instability: An introduction. $22^{\text {nd }}$ edition, Pergamon Press, New York, 1977. 